\title{
Simulation and Experimental Assembly of DNA-Graft Copolymer Micelles with Controlled Morphology
}

\author{
Zonghui Wei, ${ }^{\dagger,}$ " Yong Ren, ${ }^{\dagger},, \perp$ John-Michael Williford, ${ }^{\#}$ Wei Qu," Kevin Huang, $\square$ Shirley Ng,
} Hai-Quan Mao, ${ }^{,}, \perp$ and Erik Luijten ${ }^{*, l, O, \triangle}$

${ }^{\ddagger}$ Graduate Program in Applied Physics, "Department of Materials Science and Engineering, ${ }^{\circ}$ Department of Engineering Sciences and Applied Mathematics, and $\triangle$ Department of Physics and Astronomy, Northwestern University, Evanston, Illinois 60208, United States

${ }^{\S}$ Department of Materials Science and Engineering, Whiting School of Engineering, and $\square$ Department of Chemical and Biomolecular Engineering, Johns Hopkins University, Baltimore, Maryland 21218, United States

${ }^{\perp}$ Translational Tissue Engineering Center and Whitaker Biomedical Engineering Institute, Johns Hopkins School of Medicine, Baltimore, Maryland 21287, United States

\# Department of Biomedical Engineering, Johns Hopkins School of Medicine, Baltimore, Maryland 21205, United States

\section{Supporting Information}

\begin{abstract}
Nanoparticles formed through complexation of plasmid DNA and copolymers are promising gene-delivery vectors, offering a wide range of advantages over alternative delivery strategies. Notably, recent research has shown that the shape of these particles can be tuned, which makes it possible to gain understanding of their shape-dependent transfection properties. Whereas earlier methods achieved shape tuning through the use of block copolymers and variation of solvent polarity, here we demonstrate through a combined experimental and computational approach that the same degree of shape control can be achieved through the use of graft copolymers that are easier to synthesize and provide a wider range of parameters for shape control. Moreover, the approach presented here does not require the use of organic solvents. The simulation work provides insight into the mechanism governing the shape variation as well as an effective model to guide further design of nonviral gene-delivery vectors. Our experimental findings offer important opportunities for the facile and large-scale synthesis of biocompatible gene-delivery vectors with well-controlled shape and tunable transfection properties. The in vitro study shows that both micelle shape and transfection efficiency are strongly correlated with the key structural parameters of the graft copolymer carriers.
\end{abstract}

KEYWORDS: shape control, micelles, DNA packaging, self-assembly, transfection

\section{INTRODUCTION}

Nanoparticle-mediated delivery of plasmid DNA remains a popular nonviral gene delivery strategy that avoids the potential immunogenic and mutagenic risks associated with viral gene vectors. $^{1-3}$ Condensation of plasmid DNA with cationic polymers has been widely used to prepare nanoparticles with sizes ranging from tens to hundreds of nanometers. ${ }^{1}$ Polyelectrolyte micelles formed through complexation of DNA and polycation-polyethylene glycol (PEG) copolymers have been a popular delivery strategy to improve nanoparticle stability in biological media. ${ }^{4,5}$ In addition, advances in nanoparticle engineering have shown that it is feasible to prepare particles with distinct shapes, a property that significantly affects the biological response to nanoparticles at systemic, tissue, and cellular levels. ${ }^{6,7}$ For example, nanoparticle shape can influence their circulation time, tissue distribution, and cell binding and internalization. ${ }^{8-14}$ None of these studies, however, have pursued shape control of nanoparticles created via condensation of plasmid DNA. Moreover, whereas some studies have reported the ability to generate DNA-containing nanoparticles with nonspherical shapes and demonstrated that nanoparticle shape can influence circulation stability, cell uptake, and intracellular trafficking, ${ }^{15,16}$ those studies lacked the ability to generate a range of uniform, distinct shapes, as required for the systematic study of the role of nanoparticle shape in plasmid DNA delivery efficiency.

We recently reported a new method for controlling and tuning the shape of plasmid DNA nanoparticles that relies on variation of solvent polarity in the condensation of DNA with polyphosphoramidate- $b$-polyethylene glycol (PPA- $b$-PEG) block copolymer. ${ }^{17}$ Using a reversible cross-linking strategy to preserve the shape of nanoparticles when transferring them to aqueous media, we demonstrated that the transfection efficiency of these particles varied by more than 3 orders of

Received: February 14, 2015

Accepted: April 7, 2015

Published: April 7, 2015 
magnitude as a function of morphology. However, although we achieved a high level of shape control of PPA- $b$-PEG/DNA micelles, this approach poses certain limitations. Notably, PPA$b$-PEG block copolymers are more difficult to synthesize and can exhibit higher batch-to-batch variation, which limits their further applications.

Here, we report the formation of DNA micelles with tunable shape through complexation with grafted copolymers instead of block copolymers. Graft copolymers have been widely used in nanoparticle-mediated gene therapy, demonstrating effective DNA delivery both in vitro and in vivo. ${ }^{2,18-21}$ Nevertheless, the key factors that control the shape of DNA nanoparticles prepared with graft copolymers have not been examined. Compared to block copolymers, grafted copolymers are significantly easier to synthesize. Notably, for graft copolymers one can take advantage of the large number of reaction sites along the backbone, as opposed to block copolymers, where reactions can only take place at the ends of the blocks. ${ }^{22}$ Synthesis of block copolymers requires either initiation of polymerization from the end of one block ${ }^{23}$ or design of a conjugation site for the coupling of two blocks. ${ }^{24}$ Grafted copolymers also allow additional variation of the molecular architecture via the grafting density, thus expanding the parameter space for shape control of DNA-containing nanoparticles. Indeed, we demonstrate that by tuning grafting density and molecular weight of the PEG chain, we can systematically vary nanoparticle shape without the use of organic solvents, eliminating the need for additional crosslinking steps to preserve nanoparticle shape in aqueous conditions. The omission of cross-linking can have important advantages in facilitating the intracellular DNA release.

\section{EXPERIMENTAL SECTION}

1. Materials. Carbon tetrachloride $\left(\mathrm{CCl}_{4}\right), \mathrm{N}, \mathrm{N}$-dimethylformamide (DMF), dichloromethane (DCM), triethylamine (TEA), dipropyltriamine (DPA), triisobutylaluminum (TIBA), and all other reagents were purchased from Sigma-Aldrich (St. Louis, MO), unless otherwise specified. $N^{1}, N^{9}$-bis(trifluoroacetyl)dipropyltriamine (TFADPA) was synthesized according to the procedure reported by O'Sullivan et al. ${ }^{25}$ The cyclic monomer 4-methyl-2-oxo-2-hydro-1,3,2dioxaphospholane was prepared as reported previously. ${ }^{26} \mathrm{~N}$-hydroxysuccinimidyl ester of methoxy polyethylene glycol hexanoic acid (PEG-NHS) was purchased from NOF America Corporation (White Plains, NY).

2. Synthesis and Characterization of Polyphosphoramidate (PPA) Polymers. The synthesis of PPAs with DPA side chains, hereafter termed PPAs, was reported previously. ${ }^{27}$ Glassware was heatdried under vacuum and purged with nitrogen. Triisobutylaluminum $(1 \mathrm{~mol} / \mathrm{L}$ in hexane, $0.2 \mathrm{~mL})$ was added into $20 \mathrm{~mL}$ of anhydrous dichloromethane in a $250 \mathrm{~mL}$ round-bottom flask. Monomer (4-alkyl2-oxo-2-hydro-1,3,2-dioxaphospholane, $5.4 \mathrm{~g}$ ) was injected into initiator solution under an ice-water bath. The reaction mixture was stirred at $0{ }^{\circ} \mathrm{C}$ for $48 \mathrm{~h}$. Solvent was removed under vacuum at room temperature. The obtained polymers were dissolved in $40 \mathrm{~mL}$ of anhydrous DMF under nitrogen. TFA-DPA (37.8 g), TEA $(48 \mathrm{~mL})$, and anhydrous $\mathrm{CCl}_{4}(33 \mathrm{~mL})$ were added to polymer consequently. The mixture was stirred at $0{ }^{\circ} \mathrm{C}$ for $30 \mathrm{~min}$, then at room temperature for $24 \mathrm{~h}$. The reaction mixture was filtered and precipitated into ether. The precipitation was dried under vacuum, suspended in $20 \mathrm{~mL}$ of $25 \%$ ammonia solution, and stirred at $60{ }^{\circ} \mathrm{C}$ overnight. The solution was concentrated and dialyzed (MWCO 3500, Spectrapor, Spectrum Laboratories, CA) against deionized (DI) water for 2 days with frequent water change. PPA was obtained by lyophilization (yield 30$40 \%$ ). The MW of PPA was characterized by gel permeation chromatography (GPC) with a multiangle light-scattering detector
(MiniDawn, Wyatt Technology, Santa Barbara, CA). A $d n / d c$ value of 1.4 was used.

3. Synthesis of PPA-g-PEG Copolymers. The typical synthesis protocol of PPA-g-PEG copolymer was as follows. PPA polymer ( 8.2 $\mathrm{mg}, 0.1 \mathrm{mmol}$ of amine) was dissolved in $1 \mathrm{~mL}$ of DI water. The solution was then mixed with $20 \mathrm{mg}$ of PEG-NHS (MW $5 \mathrm{kDa}$ ) and incubated overnight. The reaction mixture was dialyzed against DI water and lyophilized to produce a white foam-like solid with a $95 \%$ yield. This protocol yielded PPA-g-PEG copolymer with a grafting density of $4 \%$ (molar percentage of PEG chains compared to the total amount of amine groups in PPA). All other PPA-g-PEG copolymers were synthesized according to the same protocol. The molecular weight of the graft copolymer was characterized by GPC with a multiangle light-scattering detector.

4. Preparation of PPA/DNA Nanoparticles. For a typical preparation of PPA-g-PEG/DNA micelles, VR1255 plasmid DNA solution $(100 \mu \mathrm{L})$ in DI water at a concentration of $100 \mu \mathrm{g} / \mathrm{mL}$ was added to an equal volume of PPA-g-PEG solution in DI water at a concentration that corresponds to a charge ratio (ratio of the total net positive charge on the PPA chains to the total negative charge on the DNA) of 5. This charge ratio was chosen after preliminary screening showed that the transfection efficiency in vitro reached a plateau at a charge ratio of 5 . The mixture was vortexed for $20 \mathrm{~s}$ and incubated for $30 \mathrm{~min}$ at room temperature.

5. Transmission Electron Microscopy. Ten microliters of PPA$g$-PEG micelles were added to an ionized 400-mesh nickel Formvar/ Carbon film TEM grid (Electron Microscope Sciences, Hatfield, PA) and incubated for $10 \mathrm{~min}$ at room temperature. The excess solution was removed and the grid was further stained with $0.2 \mu \mathrm{L}$ of uranyl acetate ( $2 \%$ solution) and air-dried for $20 \mathrm{~min}$ before imaging. TEM images were obtained with a Philips EM 420 transmission electron microscope at $120 \mathrm{kV}$.

6. In Vitro Gene Transfection. Human cervical cancer line HeLa cells (ATCC) were maintained in Dulbecco's Modified Eagle's Medium supplemented with $10 \%$ fetal bovine serum at $37{ }^{\circ} \mathrm{C}$ and $5 \% \mathrm{CO}_{2}$. Cells were seeded in 48 -well plates at a density of $2 \times 10^{4}$ cells/well and incubated overnight prior to further use. Micelles with different shapes were added to each well at a dose of $1 \mu \mathrm{g}$ of plasmid DNA. After $4 \mathrm{~h}$ of incubation, the culture media were refreshed. The culture media were removed after $48 \mathrm{~h}$ and cells were lysed with 100 $\mu \mathrm{L}$ of reporter lysis buffer for each well (Promega, Madison, WI), and subjected to two freeze-thaw cycles. Twenty $\mu \mathrm{L}$ of cell lysate supernatant was mixed with $100 \mu \mathrm{L}$ of luciferase substrate (Promega), and the light units were measured on a luminometer (20/20n Single Tube luminometer, Turner BioSystems, Sunnyvale, CA). The luciferase activity was converted to the amount of luciferase using recombinant luciferase (Promega) as the standard, and normalized against protein content using the BCA protein assay (Thermo Scientific, Rockford, IL)

7. Simulation Methods. To investigate the mechanism underlying the shape variation of PPA-g-PEG/DNA micellar nanoparticles, we perform molecular dynamics (MD) simulations of a coarse-grained bead-spring polymer model. In this model, both DNA and PPA-gPEG copolymers beads are bonded via a harmonic bond potential

$$
U_{\text {bond }}=200 \varepsilon\left(r-r_{0}\right)^{2}
$$

where $r$ is the center-to-center distance between two beads and $r_{0}=$ $2^{1 / 6} \sigma$, with $\sigma$ the Lennard-Jones (LJ) unit of length and $\varepsilon$ the LJ unit of energy.

In view of computational efficiency, the plasmid DNA is represented by a semiflexible ring polymer of 80 beads, each with an electrostatic valency of -1 . An additional harmonic bond-angle potential is applied to the DNA to represent its intrinsic stiffness ${ }^{28}$

$$
U_{\text {angle }}=4 \varepsilon\left(\theta-\theta_{0}\right)^{2}
$$

where $\theta$ is the angle between two adjacent bonds and $\theta_{0}=180^{\circ}$.

The PPA chain, with a total length of 6 beads, is represented as a sequence of groups of 3 beads with charges of $(+2,+2,-1)$ to mimic the characteristic charge pattern of PPA. The PEG chains are neutral 
with variable chain lengths of 10,5 , and 3 beads. The justification of the length of the copolymer chains is described in the Supporting Information. In contrast to the plasmid DNA, the copolymers are considered fully flexible. Upon grafting the PEG chains to the PPA, the corresponding charged beads in the PPA chain are not replaced, to avoid unrealistically large changes in overall PPA charge. In addition, monovalent counterions are present in the system to neutralize both DNA and PPA, without additional salt. All components are placed in a periodic cubic box of linear size $100 \sigma$.

All simulations are performed in an implicit solvent using a Langevin thermostat with damping time $100 \tau$, where $\tau$ is the $\mathrm{LJ}$ unit of time

$$
\tau=\sqrt{\frac{m \sigma^{2}}{\varepsilon}}
$$

in which $m$ is the LJ unit of mass. The temperature is controlled by the Langevin thermostat as well, and set to $T=1.2 \varepsilon / k_{\mathrm{B}}$, where $k_{\mathrm{B}}$ is Boltzmann's constant. Following ref 29 we set the Bjerrum length of the aqueous solvent to $3 \sigma$. The equations of motion are integrated using the velocity-Verlet algorithm.

In this implicit-solvent representation, we use LJ potentials to describe the pair interactions involved in the system. All the components except DNA interact via a purely repulsive LJ potential with cutoff at $2^{1 / 6} \sigma$, shifted to eliminate the discontinuity at the cutoff. For DNA, the pair potential is cut off at $2.5 \sigma$ (also shifted to eliminate the discontinuity). This attraction (equal to $k_{\mathrm{B}} T$ ) is necessitated by the intrinsic stiffness of the plasmid DNA, which interferes with the condensation of the relatively short chains employed in the model. Electrostatic interactions are accounted for through Ewald summation $^{30-32}$ via the particle-particle particle-mesh algorithm with a relative accuracy of $1 \times 10^{-4}$. Details of the equilibration of the simulations as well as the sampling are described in the Supporting Information.

Each simulation covers a sampling period of $1.44 \times 10^{6} \tau$ with a time step of $0.012 \tau$. For each parameter choice, 15 independent runs are performed, corresponding to 225000 independent samples.

The coarse-grained nature of the simulations precludes a quantitative comparison to experimental parameters. In exchange, this modeling strategy makes it possible to cover the large number of polymers and long time scales that are involved in the complexation process and that are inaccessible in all-atom simulations. Once the model has been calibrated by choosing only a small number of parameters to match experimental observations, the simulations can yield explicit trends and provide mechanistic insight.

\section{RESULTS AND DISCUSSION}

PPA-g-PEG graft copolymers (Figure 1a) were synthesized by reacting PEG $N$-hydroxysuccinimide active esters with different MWs to primary amines on the PPA segment with different MWs. We define the grafting density as the number of PEG chains divided by the average number of positive charges on the PPA in each copolymer. PPA-g-PEG/DNA micelles prepared with varying PEG grafting densities exhibited distinctively different morphologies (Figure $1 \mathrm{~b}-\mathrm{e}$ ). At a charge ratio of 5 , DNA-containing nanoparticles prepared with PPA homopolymer showed a condensed morphology with an average length of $73.2 \pm 23.6 \mathrm{~nm}$ and an average width of $48.9 \pm 19.0 \mathrm{~nm}$ (Figure $1 \mathrm{~b}$ ). When PEG5k (MW $5 \mathrm{kDa}$ ) was grafted onto the PPA backbone, the morphology of polymer/DNA micelles became more elongated (Figure $1 \mathrm{c}-\mathrm{e}$ ). At a PEG5k grafting density of $1 \%$ (Figure 1c), the nanoparticle length increased to $109.5 \pm 37.3 \mathrm{~nm}$ and the width decreased to $39.7 \pm 8.3 \mathrm{~nm}$. Accordingly, the aspect ratio increased from 1.57 to 2.83 , confirming the increase in anisotropy. At a PEG5k grafting density of $2 \%$ (Figure 1d), the micelles elongated further. The length and aspect ratio increased to $164.2 \pm 70.4 \mathrm{~nm}$ and 7.46

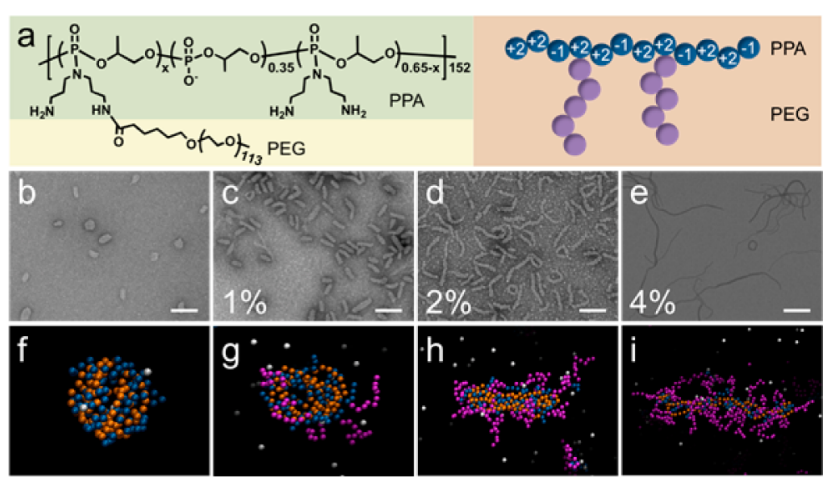

Figure 1. (a) Structure of PPA25k-g-PEG5k and schematic illustration of its coarse-grained bead-spring representation in simulation. (b) TEM images of PPA/DNA nanoparticles and PPA25k-g-PEG5k/DNA micelles at a PEG grafting density of (c) 1, (d) 2, and (e) 4\%. Scale bar $200 \mathrm{~nm}$. (f) Representative simulation configurations of PPA/DNA nanoparticles and PPA- $g$-PEG/DNA micelles at a PEG grafting density of (g) 1, (h) 2, and (i) 4\%. DNA, PPA, and PEG beads are represented in orange, blue, and purple, respectively. Monovalent ions are depicted in white.

\pm 3.86 , respectively, while the width decreased to $24.2 \pm 10.7$ $\mathrm{nm}$. When the PEG5k grafting density was increased to $4 \%$ (Figure 1e), the micelles became even longer and narrower, with a fraction of micelles exhibiting lengths around $1 \mu \mathrm{m}$. At this condition, we also observed a small population of ringshaped particles, a phenomenon found previously for PPA- $b$ PEG block copolymers. ${ }^{17}$ In general, with increasing grafting density of PEG5k the length and aspect ratio of micelles increased, their width decreased, and their morphologies became more elongated.

In simulation, plasmid DNA is represented by an anionic ring polyelectrolyte and PPA-g-PEG by a graft copolymer composed of a cationic PPA chain and neutral PEG chains of length 10 (Figure 1a). Specifically, we find that the morphologies observed at $2 \%$ grafting density occur in simulation when one PEG chain is grafted to the center of each PPA chain. Correspondingly, grafting density $4 \%$ is then represented by grafting one PEG chain to each half of the PPA chain, whereas for grafting density $1 \%$ we adopt a statistical interpretation, grafting a PEG chain to half of all PPA chains. We observe copolymer/DNA condensation for all grafting densities, including nongrafted PPA, as illustrated in Figure 1f-i. Micelles formed with PPA homopolymers display a compact, spherical shape, in accordance with the experimental observations. With increasing grafting density, the shape of the micelles changes from compact to slightly elongated, and subsequently to wormlike. This systematic elongation fully confirms the variation observed in experiment (Figure $1 \mathrm{~b}-\mathrm{e}$ ). Moreover, the similarity of these shapes to those found for DNA-block copolymer nanoparticles ${ }^{17}$ in different solvent mixtures indicates that it is possible to achieve shape control solely through variation of PEG grafting density.

The MD simulations not only reproduce the trend in micelle morphology as a function of PEG grafting density, but also provide detailed information about shape fluctuations as well as the relative probability of coexisting morphologies at different grafting densities. To quantitatively characterize nanoparticle shape, we employ the asphericity $A^{33,34}$ 


$$
A=\frac{1}{2}\left\langle\frac{\left(\lambda_{1}-\lambda_{2}\right)^{2}+\left(\lambda_{2}-\lambda_{3}\right)^{2}+\left(\lambda_{3}-\lambda_{1}\right)^{2}}{\left(\lambda_{1}+\lambda_{2}+\lambda_{3}\right)^{2}}\right\rangle
$$

where $\lambda_{1}, \lambda_{2}$, and $\lambda_{3}$ are the eigenvalues of the DNA radius-ofgyration tensor (defined in the Supporting Information). Frequency distributions of the asphericity, which takes values ranging from 0 (sphere) to 1 (straight rod), for different grafting densities are shown in Figure 2. The asphericity peak

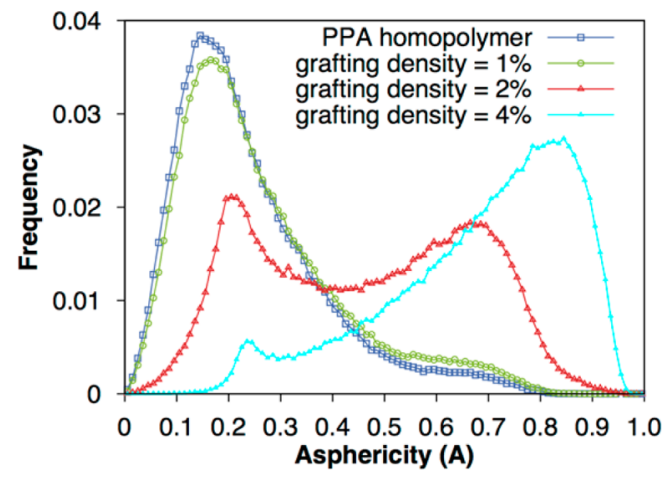

Figure 2. Frequency distribution of the asphericity of DNA contained within PPA-g-PEG/DNA micelles at different grafting densities, for our model with fixed PEG length $(N=10)$.

near $A=0.15$ reflects the near-spherical micelles observed for PPA without any grafted PEG. As the grafting density is increased to $2 \%$, the distribution takes a bimodal form, signaling coexistence between compact $(A \approx 0.2)$ and elongated $(A \approx 0.7)$ shapes. Eventually, at the highest grafting density, worm-like micelles dominate $(A \approx 0.8)$. The broad peak at grafting density $4 \%$ arises due to the flexibility of these elongated morphologies, which in turn may contribute to their transport kinetics and transfection properties. ${ }^{17}$ An interesting side note concerns the small effect of the PEG side chains at grafting density $1 \%$. This is partly due to the fact that a small number of PEG chains can be accommodated in a compact micelle without significantly affecting its free energy, but also partly due to our model choice, in which only $50 \%$ of all copolymers is grafted to account for the fact that our PPA chain is too short to generate a uniform copolymer with $1 \%$ grafting density. Indeed, we found that the nongrafted copolymers were greatly favored in the complexation process, making up $73.6 \pm$ $0.4 \%$ of the copolymers in the micelle, which in turn explains the small effect of the PEG grafting on the micelle shape.

Since our model has been proven to provide qualitative guidance, we employ additional MD simulations to probe the role of PEG chain length in controlling micelle morphology. We first perform simulations in which the PEG chain length is successively decreased from 10 to 5 and 3 beads. Figure 3 shows how at grafting densities of 2 and $4 \%$ this length reduction results in a systematic trend toward more compact micelles, indicating that the shape distribution can be fine-tuned through a combination of grafting density and PEG chain length. The lack of shape variation at grafting density $1 \%$ mirrors the observations in Figure 2.

Armed with this guidance, we performed experiments with PPA-g-PEG copolymers in which the PEG chain length was reduced from $5 \mathrm{kDa}$ to $2 \mathrm{kDa}$ and $0.8 \mathrm{kDa}$. As illustrated in Figure $4 \mathrm{a}-\mathrm{i}$, for each PEG length, the micelles became more
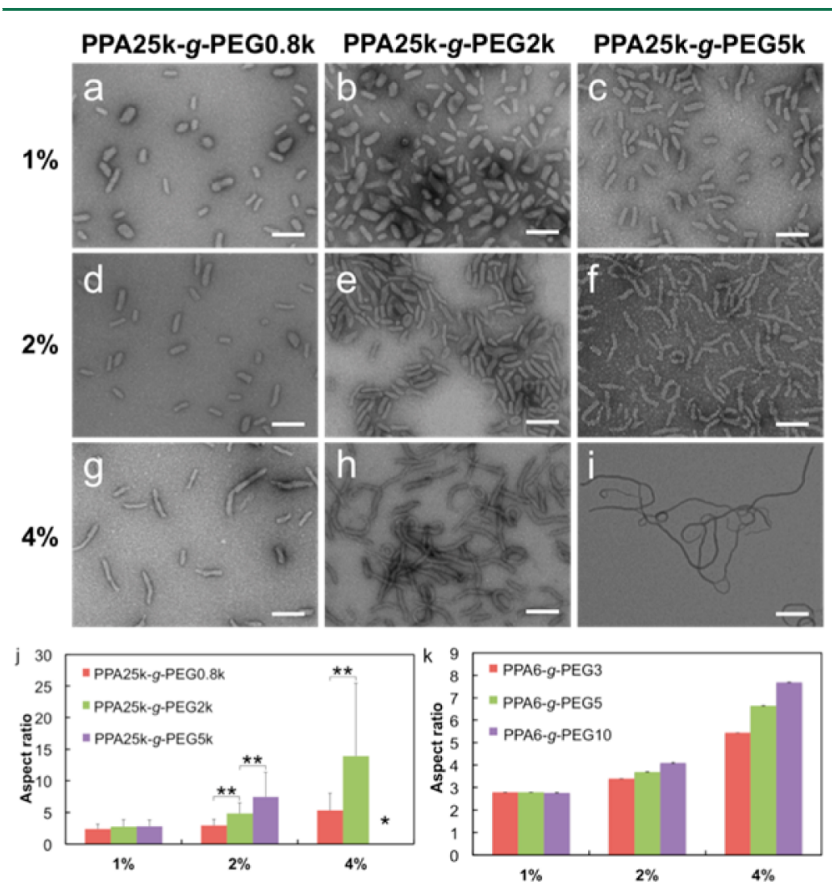

Figure 4. $(\mathrm{a}-\mathrm{i})$ TEM images of PPA25k-g-PEG/DNA micelles for PEG MW of $0.8,2$, and $5 \mathrm{kDa}$, at PEG grafting densities of $1 \%, 2 \%$, and $4 \%$. Scale bars $200 \mathrm{~nm}$. (j) Aspect ratio of the micelles determined from TEM images. Plot shows mean and standard deviation $(n>100$, ${ }^{* *} p<0.01$; Student's $t$-test). *Aspect ratio of PPA- $g$-PEG5k-4\%/DNA was not comparable with other data due to severe chain entanglement. (k) Aspect ratio of DNA in simulation. Here, each bar shows the mean and standard deviation of 225000 independent samples.

elongated as we increased the grafting density, consistent with the findings for PEG5k (Figure 1) and the modeling
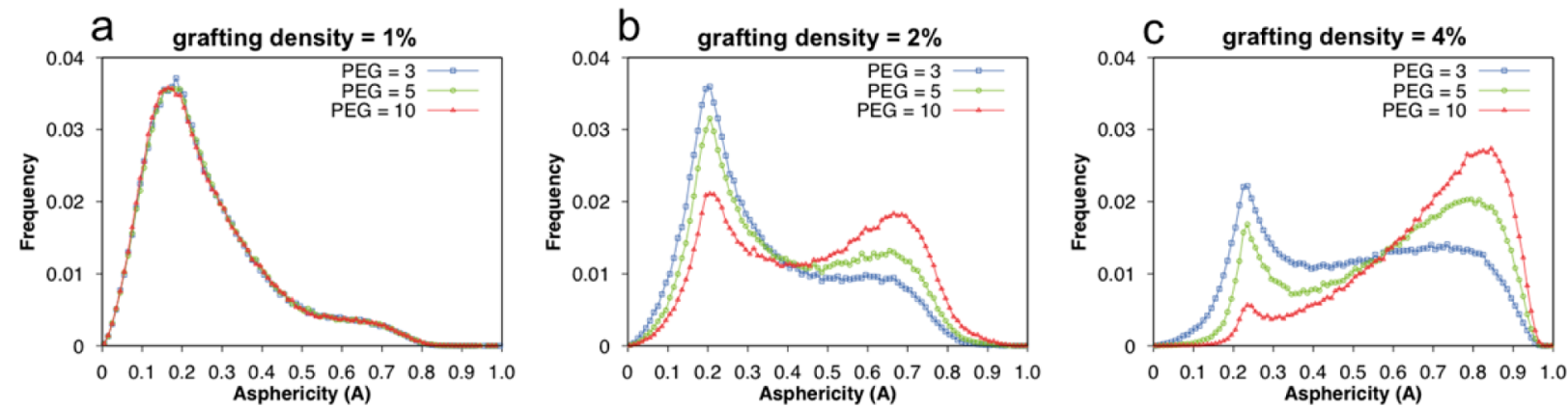

Figure 3. Frequency distribution of the asphericity of DNA within PPA-g-PEG/DNA micelles for different PEG chain lengths at three grafting densities, as obtained in molecular dynamics simulations. 
predictions (Figure 3). Conversely, we also observed systematic trends as a function of PEG degree of polymerization, for each grafting density. At a grafting density of $1 \%$, the effect on micelle shape was minimal, in accordance with the modeling prediction, but at higher grafting densities the micelles exhibited a strong trend toward elongation as the PEG chain length was varied from 0.8 to $5 \mathrm{kDa}$. To quantify these observations, we determined the micelle aspect ratio from the TEM images (Figure 4j), confirming the elongation with increasing PEG length at grafting densities of 2 and $4 \%$. Because of severe chain entanglement and difficulties in obtaining clear staining patterns for long, thin micelles from the TEM samples, the aspect ratio for PPA-g-PEG5k-4\%/DNA micelles was not determined. Nevertheless, Figure $4 \mathrm{i}$ confirms that the aspect ratio of these long and thin worms was significantly higher than in all other samples. For comparison, Figure $4 \mathrm{k}$ shows the average aspect ratio of DNA in simulation. These data confirm the agreement between simulation and experiment for the effect of grafting density as well as PEG length. For elongated shapes, the aspect ratio in simulation is smaller than the corresponding experimental values because of the shorter DNA length employed in simulation, which was chosen for computational efficiency.

The shape variations observed as a function of grafting density and PEG chain length can be interpreted and explained within a mean-field theoretical framework ${ }^{17,28}$ that incorporates the bending rigidity of the DNA, electrostatic binding between DNA and PPA, and entropy of the PEG chains. If $n$ denotes the number of PEG chains of length $N_{\mathrm{PEG}}$ in the micelle, worm-like shapes are favored if $n^{7 / 5} N_{\mathrm{PEG}}^{1 / 5} \gtrsim \Delta U_{\text {core }} / \gamma$, where $\Delta U_{\text {core }}$ is the internal-energy difference of the micelle core between elongated and spherical micelles, and $\gamma$ is a positive constant. ${ }^{28}$ Thus, this condition, which results from free-energy minimization of neutral micelles, provides an explicit predictive tool. Longer PEG chains (larger $N_{\mathrm{PEG}}$ ) or higher grafting density (larger $n$ ) favor elongated shapes. Moreover, the weak dependence of this criterion on $N_{\mathrm{PEG}}$ (exponent $1 / 5$ ) explains why an increase of PEG chain length has such a limited effect at low grafting density, precisely as observed in Figure $4 a-c$. Ultimately, at sufficiently high grafting densities, an increase of $N_{\mathrm{PEG}}$ indeed leads to elongation of the micelle shapes, as found in Figure $4 d-f$ and $4 g-i$, as well as in the simulation results in Figure $3 \mathrm{~b}$, c. In summary, the competition between the minimization of the core energy of the nanoparticles (which depends on a combination of DNA bending rigidity, electrostatic complexation between DNA and PPA, and interactions of both DNA and PPA with the aqueous solvent) and maximization of the entropy of the corona controls their shape. As demonstrated, variation of the corona entropy by changing either the grafting density or the length of the PEG chains makes it possible to systematically vary the shape from spherical to elongated.

In view of the transfection studies performed with DNA micelles based upon block copolymers, ${ }^{17}$ it is meaningful to compare their morphology to micelles formed through complexation with grafted copolymers. Figure 5 illustrates the asphericity distribution obtained in MD simulation of block and graft copolymers with PPA chains of length 6 and one PEG chain of length 10 . Whereas the overall shape distribution for both systems is very similar, with a bimodal character reflecting the coexistence of compact and elongated micelles, the grafted copolymers yield slightly more elongated micelles. This difference is statistically significant, with an average DNA

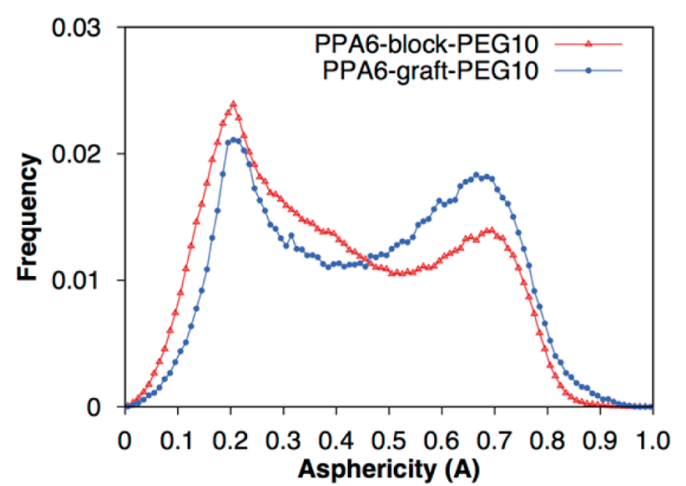

Figure 5. Frequency distributions of the asphericity of DNA contained within DNA/PPA- $b$-PEG micelles and PPA-g-PEG/DNA micelles. Micelles based upon grafted copolymers display a small but statistically significant bias toward more elongated shapes.

asphericity of $0.406 \pm 0.002$ for block copolymers and $0.462 \pm$ 0.002 for graft copolymers. A possible explanation for this difference lies in the manner in which the PEG chains are positioned within the micelle. For the block copolymer, PEG is linked to the bead with charge -1 at one end of the PPA chain. Upon complexation with the anionic DNA, the repulsion between this PPA bead and the DNA tends to displace the PEG chain away from the micelle core. In contrast, for the grafted case, the PEG is linked to the +2 bead of the PPA in the center of the PPA chain. The electrostatic attraction between this PPA bead and the DNA bead significantly restricts the motion of PEG chain, which in turn leads to more elongated shapes of the micelle core.

To investigate the effect of polymer structure and micelle shape on transfection efficiency, we transfected HeLa cells with a series of micelles prepared with graft copolymer carriers containing different MW PEG grafts $(0.8,2,5,10 \mathrm{kDa})$, different MWs of PPA chains $(12,25,65 \mathrm{kDa})$, or different PEG grafting densities $(1,2,3$, and 4\%). TEM images of the micelles prepared with these graft copolymers are shown in Figure S2. It is also important to point out that these micellar nanoparticles exhibit high serum stability when incubated in serum-containing medium (Figure S3). Figure 6a shows that at fixed PPA MW (25 kDa) and PEG MW, transfection decreased as grafting density increased. At fixed PPA MW and grafting density, transfection efficiency decreased as PEG MW

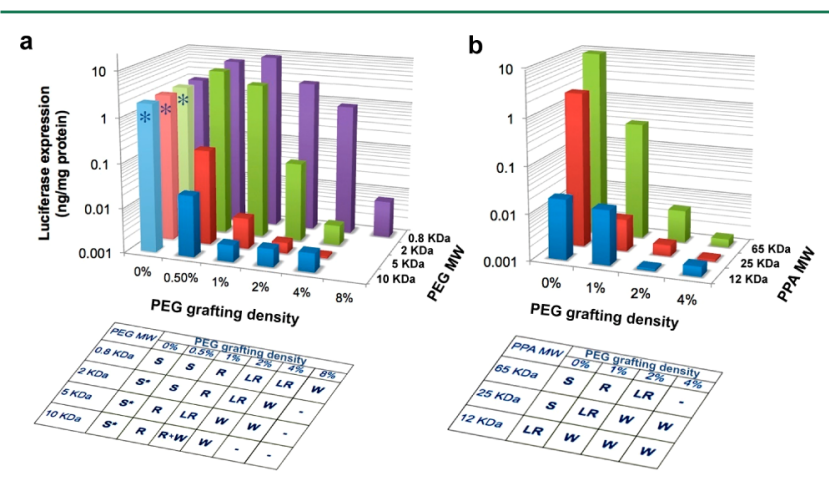

Figure 6. Transfection efficiency and shapes of micelles prepared from PPA-g-PEG copolymers. (a) PPA MW $25 \mathrm{kDa}$, different PEG MWs, and different PEG grafting densities. (b) PEG MW $5 \mathrm{kDa}$, different PPA MWs, and different PEG grafting densities. * At 0\% PEG grafting density, the particles are all the same. S, spheres; R, rods; LR, long rods, $\mathrm{W}$, worms. 
increased. Figure $6 \mathrm{~b}$ shows that at fixed PEG MW and grafting density, transfection efficiency increased as the MW of PPA increased. From the tables of micelle shape in Figure 6a, b, we observed that the trend of micelle shape correlated very well with that of transfection efficiency. Micelles with more elongated shape tended to yield decreased transfection efficiency. Generally, with more PEG grafted on the backbone, on average more elongated shapes and lower transfection efficiencies were found.

To compare the transfection efficiency and shape of micelles prepared from copolymers with different MW PEG grafts, PPA backbone MW, and PEG grafting densities, we propose the DNA compaction factor as an effective combination of these three factors

$$
\begin{aligned}
& \text { DNA compaction factor } \\
& =\frac{\text { PEG grafting density } \times \text { PEG chain length }}{\text { PPA charge number }} \times 10^{4}
\end{aligned}
$$

where PEG chain length is the average number of PEG repeat units per graft, PPA charge number is the average number of positive charges in each copolymer, and the factor 10000 is included to facilitate comparison of the numerical values. Note that, since the grafting density is defined as the number of grafted PEG chains divided by PPA charge number, the compaction factor increases linearly with the number of PEG units per copolymer, but decreases quadratically with the copolymer charge. Since both a higher amount of positive charge and a lower number of PEG units favor the compaction of DNA, a lower Compaction Factor provides a heuristic indicator for stronger DNA condensation and a more compact shape. Indeed, Figure 7 combines the results of all micelles prepared with different graft copolymers as described in Figure 6 and Figure S2 confirming the strong correlation between the transfection efficiency and Compaction Factor of various copolymer carriers. For all PEG and PPA MWs and PEG grafting densities studied, for a Compaction Factor below 20, all micelles exhibited a condensed spherical shape and showed high transfection efficiency above $4 \mathrm{ng} / \mathrm{mg}$ luciferase expression. When the Compaction Factor was in the range between 20 and 50, most of the micelles showed a slightly elongated short rod-like shape, and their transfection efficiencies fell to the range of 4 to $0.1 \mathrm{ng} / \mathrm{mg}$. With the Compaction Factor in the range of 50 to 100, the micelles become more elongated and occasionally wormlike, and all those micelles showed transfection efficiency in the range of 100 to $4 \mathrm{pg} / \mathrm{mg}$. When the Compaction Factor was larger than 100 , most micelles exhibited long and thin worm-like shapes, with all but one of the experiments resulting in transfection efficiencies below $4 \mathrm{pg} / \mathrm{mg}$. It is apparent that the average transfection efficiencies of micelles with different shapes decrease systematically according to the sequence of spheres, short rods, long rods, and worms (Figure S4). Thus, all data confirm that the transfection efficiency correlates well with micelle shape, with more condensed shapes generally corresponding to higher transfection efficiency. The effect of shape on the transfection efficiency of these micellar nanoparticles may be related to cellular binding and uptake activities and their colloidal stability. Our previous studies on nanoparticles prepared with PPA- $b$-PEG carriers confirmed that wormlike particles showed significantly a lower cellular uptake level and higher stability than rodlike and spherical nano-
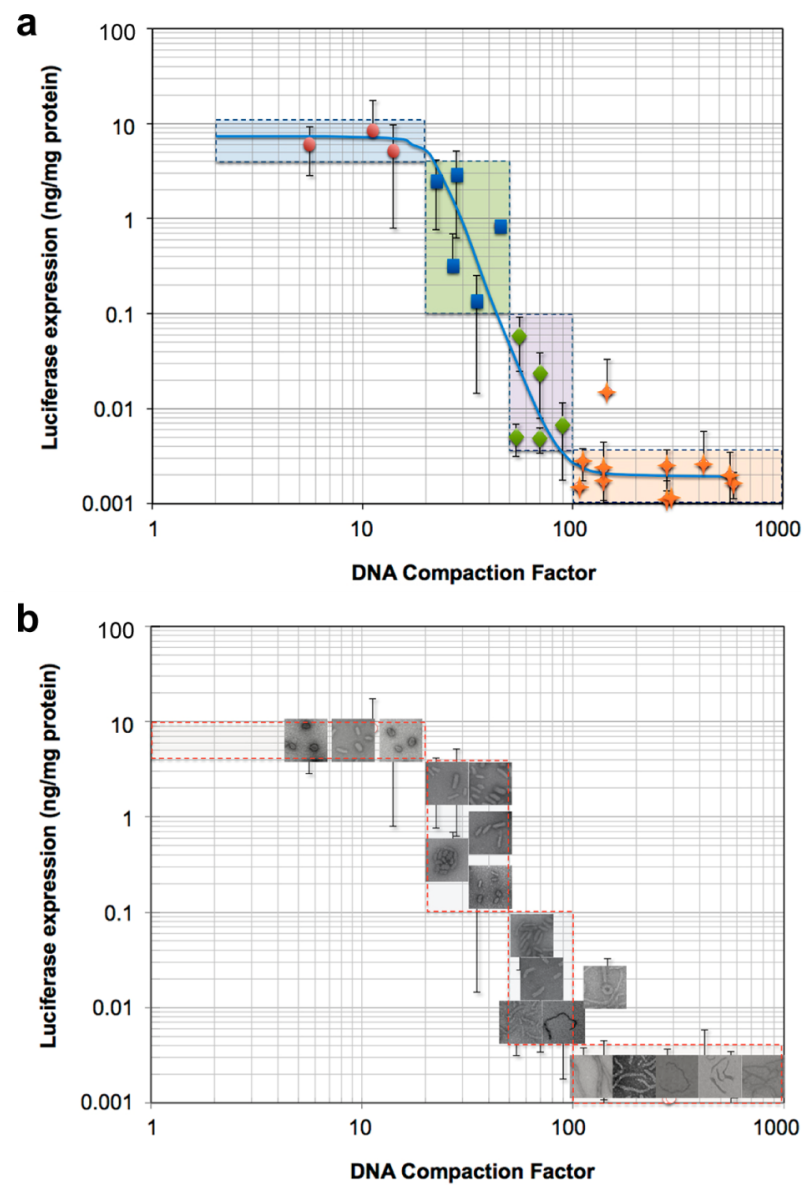

Figure 7. Transfection efficiency of micelles as a function of DNA Compaction Factor (eq 5) of PPA-g-PEG copolymers $(n=3)$. The bottom panel shows representative TEM images of the different nanoparticle shapes, ranging from spherical (small compaction factor) to elongated (large compaction factor).

particles. ${ }^{17,23}$ Besides cell uptake, nanoparticle shape may also play an important role in DNA condensation kinetics, intracellular trafficking, and DNA release kinetics of the nanoparticles, which collectively influence the transfection efficiency in vitro. The detailed mechanisms as well as the shape effect of these nanoparticles on in vivo gene-delivery efficiency remain to be elucidated.

\section{CONCLUSIONS}

In summary, through an integrated experimental and computational approach, we have systematically examined shape control of micellar nanoparticles self-assembled via complexation of plasmid DNA and PPA-g-PEG grafted copolymers. Graft copolymers not only are easier to synthesize than the block copolymers employed in earlier studies but also allow access to a wider parameter set for exploration of the structural origins of shape control in DNA micelle assembly. Shape variation was achieved in aqueous medium without the need of organic solvents and preservation of micelle shape through crosslinking. By experimental screening of several structural parameters of the copolymer carriers and by computational modeling, we demonstrated that shape control of these complex-core micelles in aqueous media is primarily governed by the condensation ability of the polycationic chain and the PEG chain entropy. When these micelles are assembled in water, we can semiquantitatively express the role of these 
factors in terms of a single "DNA compaction factor," which summarizes the trend of shape variation and closely correlates with the transfection efficiency in vitro. In addition, in this work we highlight the advantages of an integrated experimental and computational approach, which not only aids in accelerating the identification of the key structural and experimental parameters for shape variation of the DNA/copolymer micelles but also provides insights into the governing mechanism and offers a testable model to guide future carrier design for nonviral gene delivery in terms of morphology control.

\section{ASSOCIATED CONTENT}

\section{S Supporting Information}

The Supporting Information is available free of charge on the ACS Publications website at DOI: 10.1021/acsbiomaterials.5b00080.

Additional details on the simulation model and parameter choices; additional experimental results (PDF)

\section{AUTHOR INFORMATION}

\section{Corresponding Authors}

*E-mail: hmao@jhu.edu. Phone: 410-516-8792.

*E-mail: luijten@northwestern.edu. Phone: 847-491-4097.

\section{Author Contributions}

${ }^{\dagger}$ Authors Z.W. and Y.R. contributed equally. The manuscript was written through contributions of all authors. All authors have given approval to the final version of the manuscript.

Notes

The authors declare no competing financial interest.

\section{ACKNOWLEDGMENTS}

This research is supported by NSF grant DMR-1310211 (EL), NIH/NIBIB grant R21 EB013274 (HQM and EL), NIH/ NIGMS grant R01 GM073937 (HQM), and a pilot grant from the Institute for NanoBioTechnology at Johns Hopkins University. We thank the Quest high-performance computing facility at Northwestern University for computational resources.

\section{REFERENCES}

(1) Pack, D. W.; Hoffman, A. S.; Pun, S.; Stayton, P. S. Design and development of polymers for gene delivery. Nat. Rev. Drug Discovery 2005, 4, 581-593.

(2) Wong, S. Y.; Pelet, J. M.; Putnam, D. Polymer systems for gene delivery-past, present, and future. Prog. Polym. Sci. 2007, 32, 799-837.

(3) Zhang, Y.; Satterlee, A.; Huang, L. In vivo gene delivery by nonviral vectors: overcoming hurdles? Mol. Ther. 2012, 20, 12981304.

(4) Osada, K.; Christie, R. J.; Kataoka, K. Polymeric micelles from poly(ethylene glycol)-poly(amino acid) block copolymer for drug and gene delivery. J. R. Soc., Interface 2009, 6 (Suppl 3), S325-39.

(5) Bae, Y.; Kataoka, K. Intelligent polymeric micelles from functional poly(ethylene glycol)-poly(amino acid) block copolymers. Adv. Drug Delivery Rev. 2009, 61, 768-84.

(6) Albanese, A.; Tang, P. S.; Chan, W. C. The effect of nanoparticle size, shape, and surface chemistry on biological systems. Annu. Rev. Biomed. Eng. 2012, 14, 1-16.

(7) Decuzzi, P.; Pasqualini, R.; Arap, W.; Ferrari, M. Intravascular Delivery of Particulate Systems: Does Geometry Really Matter? Pharm. Res. 2009, 26, 235-243.

(8) Agarwal, R.; Singh, V.; Jurney, P.; Shi, L.; Sreenivasan, S. V.; Roy, K. Mammalian cells preferentially internalize hydrogel nanodiscs over nanorods and use shape-specific uptake mechanisms. Proc. Natl. Acad. Sci. U. S. A. 2013, 110, 17247-17252.

(9) Barua, S.; Yoo, J. W.; Kolhar, P.; Wakankar, A.; Gokarn, Y. R.; Mitragotri, S. Particle shape enhances specificity of antibody-displaying nanoparticles. Proc. Natl. Acad. Sci. U. S. A. 2013, 110, 3270-3275.

(10) Chauhan, V. P.; Popovic, Z.; Chen, O.; Cui, J.; Fukumura, D.; Bawendi, M. G.; Jain, R. K. Fluorescent Nanorods and Nanospheres for Real-Time In Vivo Probing of Nanoparticle Shape-Dependent Tumor Penetration. Angew. Chem., Int. Ed. 2011, 50, 11417-11420.

(11) Chithrani, B. D.; Ghazani, A. A.; Chan, W. C. W. Determining the size and shape dependence of gold nanoparticle uptake into mammalian cells. Nano Lett. 2006, 6, 662-668.

(12) Geng, Y.; Dalhaimer, P.; Cai, S. S.; Tsai, R.; Tewari, M.; Minko, T.; Discher, D. E. Shape effects of filaments versus spherical particles in flow and drug delivery. Nat. Nanotechnol. 2007, 2, 249-255.

(13) Gratton, S. E. A.; Ropp, P. A.; Pohlhaus, P. D.; Luft, J. C.; Madden, V. J.; Napier, M. E.; DeSimone, J. M. The effect of particle design on cellular internalization pathways. Proc. Natl. Acad. Sci. U. S. A. 2008, 105, 11613-11618.

(14) Kolhar, P.; Anselmo, A. C.; Gupta, V.; Pant, K.; Prabhakarpandian, B.; Ruoslahti, E.; Mitragotri, S. Using shape effects to target antibody-coated nanoparticles to lung and brain endothelium. Proc. Natl. Acad. Sci. U. S. A. 2013, 110, 10753-10758.

(15) Shi, J.; Choi, J. L.; Chou, B.; Johnson, R. N.; Schellinger, J. G.; Pun, S. H. Effect of polyplex morphology on cellular uptake, intracellular trafficking, and transgene expression. ACS Nano 2013, $7,10612-10620$.

(16) Tockary, T. A.; Osada, K.; Chen, Q.; Machitani, K.; Dirisala, A.; Uchida, S.; Nomoto, T.; Toh, K.; Matsumoto, Y.; Itaka, K.; Nitta, K.; Nagayama, K.; Kataoka, K. Tethered PEG crowdedness determining shape and blood circulation profile of polyplex micelle gene carriers. Macromolecules 2013, 46, 6585-6592.

(17) Jiang, X.; Qu, W.; Pan, D.; Ren, Y.; Williford, J. M.; Cui, H. G.; Luijten, E.; Mao, H. Q. Plasmid-Templated Shape Control of Condensed DNA-Block Copolymer Nanoparticles. Adv. Mater. 2013, 25, 227-232.

(18) Jiang, X.; Dai, H.; Leong, K. W.; Goh, S. H.; Mao, H. Q.; Yang, Y. Y. Chitosan-g-PEG/DNA complexes deliver gene to the rat liver via intrabiliary and intraportal infusions. J. Gene Med. 2006, 8, 477-487.

(19) Lee, M.; Kim, S. W. Polyethylene glycol-conjugated copolymers for plasmid DNA delivery. Pharm. Res. 2005, 22, 1-10.

(20) Patnaik, S.; Gupta, K. C. Novel polyethylenimine-derived nanoparticles for in vivo gene delivery. Expert Opin. Drug Delivery 2013, 10, 215-228.

(21) Petersen, H.; Fechner, P. M.; Martin, A. L.; Kunath, K.; Stolnik, S.; Roberts, C. J.; Fischer, D.; Davies, M. C.; Kissel, T. Polyethylenimine-graft-poly(ethylene glycol) copolymers: influence of copolymer block structure on DNA complexation and biological activities as gene delivery system. Bioconjugate Chem. 2002, 13, 845854.

(22) Burnett, G. M. Block and Graft Copolymers. Annu. Rev. Phys. Chem. 1959, 10, 103-122.

(23) Jiang, X.; Leong, D.; Ren, Y.; Li, Z. P.; Torbenson, M. S.; Mao, H. Q. String-Like Micellar Nanoparticles Formed by Complexation of PEG- $b$-PPA and Plasmid DNA and Their Transfection Efficiency. Pharm. Res. 2011, 28, 1317-1327.

(24) Schatz, C.; Louguet, S.; Le Meins, J. F.; Lecommandoux, S. Polysaccharide-block-polypeptide copolymer vesicles: towards synthetic viral capsids. Angew. Chem., Int. Ed. 2009, 48, 2572-2575.

(25) Osullivan, M. C.; Dalrymple, D. M. A One-Step Procedure for the Selective Trifluoroacetylation of Primary Amino-Groups of Polyamines. Tetrahedron Lett. 1995, 36, 3451-3452.

(26) Wang, J.; Zhang, P. C.; Lu, H. F.; Ma, N.; Wang, S.; Mao, H. Q.; Leong, K. W. New polyphosphoramidate with a spermidine side chain as a gene carrier. J. Controlled Release 2002, 83, 157-168.

(27) Ren, Y.; Jiang, X. A.; Pan, D.; Mao, H. Q. Charge Density and Molecular Weight of Polyphosphoramidate Gene Carrier Are Key Parameters Influencing Its DNA Compaction Ability and Transfection Efficiency. Biomacromolecules 2010, 11, 3432-3439. 
(28) Qu, W.; Ren, Y.; Jiang, X.; Pan, D.; Williford, J. M.; Wei, Z.; Mao, H. Q.; Luijten, E. Morphology Control of Micellar DNA/PEG-bPolycation Nanoparticles. 2015, in preparation.

(29) Stevens, M. J.; Kremer, K. The Nature of Flexible Linear Polyelectrolytes in Salt-Free Solution - a Molecular-Dynamics Study. J. Chem. Phys. 1995, 103, 1669-1690.

(30) Sanders, L. K.; Xian, W.; Guáqueta, C.; Strohman, M. J.; Vrasich, C. R.; Luijten, E.; Wong, G. C. L. Control of electrostatic interactions between F-actin and genetically modified lysozyme in aqueous media. Proc. Natl. Acad. Sci. U. S. A. 2007, 104, 15994-15999.

(31) Guáqueta, C.; Luijten, E. Polyelectrolyte Condensation Induced by Linear Cations. Phys. Rev. Lett. 2007, 99, 138302.

(32) Hsiao, P.-Y.; Luijten, E. Salt-Induced Collapse and Reexpansion of Highly Charged Flexible Polyelectrolytes. Phys. Rev. Lett. 2006, 97, 148301.

(33) Rudnick, J.; Gaspari, G. The Shapes of Random-Walks. Science 1987, 237, 384-389.

(34) Guo, L.; Luijten, E. Shape variation of linear polymers upon phase separation in a ternary solution. Macromolecules 2003, 36, $8201-8204$. 